\title{
Black holes in an expanding universe from fake supergravity
}

\author{
Samuele Chimento and Dietmar Klemm \\ Dipartimento di Fisica, Università di Milano, and \\ INFN, Sezione di Milano, \\ Via Celoria 16, 20133 Milano, Italy. \\ E-mail: samuele.chimento@mi.infn.it, dietmar.klemm@mi.infn.it
}

ABSTRACT: In arXiv:0902.4814, a general recipe to construct fake supersymmetric solutions to fake $N=2, d=4$ gauged supergravity coupled to abelian vector multiplets was presented. We use these results to find new multi-centered black hole solutions in an asymptotically FLRW universe. These satisfy the weak energy condition and are maximally charged under two U(1) gauge fields coupled to a scalar, which drives the cosmic expansion while rolling down its potential. As a special subcase, our black holes include the ones constructed previously by Gibbons and Maeda in arXiv:0912.2809. The latter contain two non-negative real numbers $n_{S}, n_{T}$ obeying the constraint $n_{S}+n_{T}=4$, with the cases $n_{T}=4$ and $n_{T}=1$ corresponding to the Kastor-Traschen and the MaedaOhta-Uzawa solution respectively. We show that $n_{S}, n_{T}$ arise directly as exponents in the prepotential of the fake supergravity theory, and that the above constraint stems from the fact that the prepotential must be a homogeneous function of degree two. Finally, some physical properties of the black holes, like asymptotic behaviour, curvature singularities and trapping horizons, are also discussed. Similar to other solutions that appeared previously in the literature, there is a symmetry enhancement near the event horizon, which becomes therefore a Killing horizon, in spite of the highly dynamical nature of the original spacetime. The temperature associated to this Killing horizon turns out to be nonvanishing.

Keywords: Black Holes, Supergravity Models, Black Holes in String Theory. 


\section{Contents}

1 Introduction $\quad 1$

2 Fake $N=2, d=4$ gauged supergravity 3

2.1 Special geometry 3

2.2 Fake Killing spinors 4

2.3 Fake supersymmetric solutions 5

3 The $\mathcal{F}(\chi)=-\frac{i}{4}\left(\chi^{0}\right)^{n}\left(\chi^{1}\right)^{2-n}$ model $\quad 6$

3.1 Construction of the solution $\quad 7$

3.2 Physical discussion 9

4 Alternative model $\quad 17$

4.1 Construction of the solution 19

$\begin{array}{lll}5 & \text { Final remarks } & 20\end{array}$

\section{Introduction}

Since the seventies of the last century, the physics of black holes has raised several fascinating problems and puzzles, whose resolution is believed to be crucial for the construction of a future quantum theory of gravity. Indeed, much of what we presently know on quantum effects in strong gravitational fields comes from the study of stationary black holes, which by now are quite well understood.

On the other hand, much less is known on dynamical processes involving black holes, since only a few time-dependent black hole solutions have been constructed so far. The first and perhaps most famous one is the McVittie spacetime [1], but there have been some controversies in the literature concerning its interpretation as a black hole embedded in an FLRW universe [2-4]. Using conformal techniques, Sultana and Dyer [5] constructed a black hole in a dynamical background, which however suffers from the violation of energy conditions.

Other notable exceptions include the Kastor-Traschen solution [6], that describes an arbitrary number of black holes in de Sitter space, each of which carrying an electric charge equal to the mass. This leads to a no-force condition, such that the whole system 
is just comoving with the cosmological expansion. Five-dimensional multi-centered rotating charged de Sitter black holes were constructed in [7, 8]. Maeda, Ohta and Uzawa (MOU in what follows) [9] used dynamical intersecting brane solutions in higher dimensions to obtain four- and five-dimensional black holes that asymptotically tend to an FLRW universe filled with stiff matter. These spacetimes were further studied in [10], and generalized to arbitrary dimension in $[11]^{1}$. Generically, the solutions in $[6,9,11]$ are given in terms of harmonic functions, such that one can superpose an arbitrary number of black holes, in spite of the lack of supersymmetry. For the five-dimensional case of [11], it was shown in [13] that this equilibrium condition can be traced back to the existence of a fake Killing spinor ${ }^{2}$. By solving the first-order fake Killing spinor equations rather than the second-order Einstein equations, the authors of [13] were then able to find a spinning generalization of their five-dimensional cosmological black hole.

In this paper, we will pick up on this idea, in order to construct new multi-centered black hole solutions in an asymptotically FLRW universe in four dimensions. To this end, we shall make essential use of the results of [15], where a general recipe to construct fake supersymmetric solutions to fake $N=2, d=4$ gauged supergravity coupled to (non)abelian vector multiplets was provided ${ }^{3}$. We shall choose models containing just one abelian vector multiplet, such that our solutions are charged under two U(1) gauge fields coupled to a scalar. As a special subcase, they include the black holes constructed previously by Gibbons and Maeda [17]. The latter contain two non-negative real numbers $n_{S}, n_{T}$ obeying the constraint $n_{S}+n_{T}=4$, with the cases $n_{T}=4$ and $n_{T}=1$ corresponding to the Kastor-Traschen and the MOU solution respectively. We show that $n_{S}, n_{T}$ arise as exponents in the prepotential of the fake supergravity theory, and the constraint $n_{S}+n_{T}=4$ (which is somehow ad hoc in [17]) simply corresponds to the requirement that the prepotential must be a homogeneous function of degree two. Moreover, our paper clarifies that the superposition principle in the Gibbons-Maeda solution is just a consequence of the existence of a fake Killing spinor. Although the matter content in the Lagrangian is the same, the multi-centered dynamical black holes constructed here are more general than the ones in [17]; for instance our scalar potential is a sum of several exponentials, which reduces to the Liouville-type potential of [17] if one of the constants characterizing the model is set to zero.

The remainder of this paper is organized as follows: In section 2 we review fake

\footnotetext{
${ }^{1}$ See also [12] for a review.

${ }^{2}$ Kastor and Traschen showed in [14] that their solution satisfies the Killing spinor equation of minimal gauged supergravity, with a Wick-rotated coupling constant, $g=i H$. In a more modern language, we would call this a fake Killing spinor equation.

${ }^{3}$ For related work in five dimensions cf. [16].
} 
$N=2, d=4$ Fayet-Iliopoulos gauged supergravity, and the recipe to construct fake supersymmetric solutions obtained in [15]. In section 3, we consider a simple prepotential that contains one vector multiplet, construct dynamical multi-centered black hole solutions to this model, and discuss in some detail their physical properties. In 4, a slightly different model is considered, and it is shown that this leads to the Gibbons-Maeda spacetime, with one of the two U(1) gauge fields dualized. We conclude in section 5 with some final remarks.

\section{$2 \quad$ Fake $N=2, d=4$ gauged supergravity}

\subsection{Special geometry}

In $N=2, d=4$ supergravity coupled to $n_{V}$ vector multiplets, the complex scalars of the multiplets parametrize an $n_{V}$-dimensional Kähler-Hodge manifold, which is the base of a symplectic bundle with the covariantly holomorphic sections ${ }^{4}$

$$
\mathcal{V}=\left(\begin{array}{c}
\mathcal{L}^{\Lambda} \\
\mathcal{M}_{\Lambda}
\end{array}\right), \quad \mathcal{D}_{\bar{\imath}} \mathcal{V} \equiv \partial_{\bar{\imath}} \mathcal{V}-\frac{1}{2}\left(\partial_{\bar{\imath}} \mathcal{K}\right) \mathcal{V}=0
$$

obeying the constraint

$$
\langle\mathcal{V}, \overline{\mathcal{V}}\rangle \equiv \overline{\mathcal{L}}^{\Lambda} \mathcal{M}_{\Lambda}-\mathcal{L}^{\Lambda} \overline{\mathcal{M}}_{\Lambda}=-i
$$

where $\mathcal{K}$ is the Kähler potential. We also introduce the explicitly holomorphic sections

$$
\Omega \equiv e^{-\mathcal{K} / 2} \mathcal{V} \equiv\left(\begin{array}{c}
\chi^{\Lambda} \\
\mathcal{F}_{\Lambda}
\end{array}\right)
$$

if the theory is defined by a prepotential $\mathcal{F}(\chi)$, then $\mathcal{F}_{\Lambda}=\partial_{\Lambda} \mathcal{F}$. In terms of the sections $\Omega$ the constraint (2.2) becomes

$$
\langle\Omega, \bar{\Omega}\rangle \equiv \bar{\chi}^{\Lambda} \mathcal{F}_{\Lambda}-\chi^{\Lambda} \overline{\mathcal{F}}_{\Lambda}=-i e^{-\mathcal{K}}
$$

The couplings of the vector fields with the scalars are determined by the matrix $\mathcal{N}$, defined by the relations

$$
\mathcal{M}_{\Lambda}=\mathcal{N}_{\Lambda \Sigma} \mathcal{L}^{\Sigma}, \quad \mathcal{D}_{\bar{\imath}} \overline{\mathcal{M}}_{\Lambda}=\mathcal{N}_{\Lambda \Sigma} \mathcal{D}_{\bar{\imath}} \overline{\mathcal{L}}^{\Sigma}
$$

In a theory with a prepotential, $\mathcal{N}$ can be obtained from

$$
\mathcal{N}_{\Lambda \Sigma}=\overline{\mathcal{F}}_{\Lambda \Sigma}+2 i \frac{\mathfrak{I m}(\mathcal{F})_{\Lambda \Lambda^{\prime}} \chi^{\Lambda^{\prime} \mathfrak{I m}(\mathcal{F})_{\Sigma \Sigma^{\prime}} \chi^{\Sigma^{\prime}}}}{\chi^{\Omega} \mathfrak{I m}(\mathcal{F})_{\Omega \Omega^{\prime}} \chi^{\Omega^{\prime}}},
$$

\footnotetext{
${ }^{4}$ Here and in what follows we use the conventions of [15].
} 
where $\mathcal{F}_{\Lambda \Sigma}=\partial_{\Lambda} \partial_{\Sigma} \mathcal{F}$.

The bosonic Lagrangian in the case of abelian vector multiplets, and with FayetIliopoulos gauging of a U(1) R-symmetry subgroup, takes the form

$$
\begin{aligned}
e^{-1} \mathcal{L}_{\text {bos }}= & R+2 \mathcal{G}_{i \bar{\jmath}} \partial_{a} Z^{i} \partial^{a} \bar{Z}^{\bar{\jmath}}-V \\
& +2 \mathfrak{I m}(\mathcal{N})_{\Lambda \Sigma} F_{a b}^{\Lambda} F^{\Sigma a b}-2 \mathfrak{R e}(\mathcal{N})_{\Lambda \Sigma} F_{a b}^{\Lambda} \star F^{\Sigma a b},
\end{aligned}
$$

where the gauging is realized with the connection $C_{\Lambda} A^{\Lambda}$ and the scalar potential is given by

$$
V=-\frac{g^{2}}{2}\left[4\left|C_{\Lambda} \mathcal{L}^{\Lambda}\right|^{2}+\frac{1}{2} \mathfrak{I m}(\mathcal{N})^{-1 \mid \Lambda \Sigma} C_{\Lambda} C_{\Sigma}\right] .
$$

Since the matrix $\mathfrak{I m}(\mathcal{N})_{\Lambda \Sigma}$ appears in the kinetic term of the vector fields, it must be negative definite and thus invertible. It can therefore be used as a 'metric' to raise and lower $\Lambda, \Sigma, \ldots$ indices.

\section{$2.2 \quad$ Fake Killing spinors}

If we perform a Wick rotation on the gauge coupling constant of the theory, $g \rightarrow i g$, we obtain a new, non-supersymmetric theory with $V \rightarrow-V$ and a gauged $\mathbb{R}$-symmetry; the Killing spinor equations, coming from the vanishing of the fermionic supersymmetry variations, become

$$
\begin{aligned}
\mathbb{D}_{a} \epsilon_{I} & =\left[-2 i \mathcal{L}_{\Lambda} F_{a b}^{\Lambda+} \gamma^{b}-\frac{i g}{4} C_{\Lambda} \mathcal{L}^{\Lambda} \gamma_{a}\right] \varepsilon_{I J} \epsilon^{J}, \\
i \not \partial Z^{i} \epsilon^{I} & =\left[\bar{f}_{\Lambda}^{i} \not^{\Lambda+}-\frac{g}{2} C_{\Lambda} \bar{f}^{i \Lambda}\right] \varepsilon^{I J} \epsilon_{J},
\end{aligned}
$$

where

$$
\mathbb{D}_{a} \epsilon_{I} \equiv\left(\nabla_{a}+\frac{i}{2} \mathcal{Q}_{a}-\frac{g}{2} C_{\Lambda} A_{a}^{\Lambda}\right) \epsilon_{I}
$$

$\mathcal{Q}_{a}=(2 i)^{-1}\left(\partial_{a} Z^{i} \partial_{i} \mathcal{K}-\partial_{a} \bar{Z}^{\bar{\imath}} \partial_{\bar{\imath}} \mathcal{K}\right)$ is the gauge field of the Kähler $\mathrm{U}(1)$, and $f_{i}^{\Lambda} \equiv$ $\mathcal{D}_{i} \mathcal{L}^{\Lambda}=\left(\partial_{i}+\frac{1}{2} \partial_{i} \mathcal{K}\right) \mathcal{L}^{\Lambda}$

Since these equations do not come from supersymmetry, they are called fake Killing spinor equations, and solutions for which they are satisfied are known as fake supersymmetric.

From the fake Killing spinors one can construct the bilinears

$$
X=\frac{1}{2} \varepsilon^{I J} \bar{\epsilon}_{I} \epsilon_{J}, \quad V_{a}=i \bar{\epsilon}^{I} \gamma_{a} \epsilon_{I}, \quad V_{a}^{x}=i\left(\sigma^{x}\right)_{I}^{J} \bar{\epsilon}^{I} \gamma_{a} \epsilon_{J}
$$

and the real symplectic sections of Kähler weight zero

$$
\mathcal{R} \equiv \mathfrak{R e}(\mathcal{V} / X), \quad \mathcal{I} \equiv \mathfrak{I m}(\mathcal{V} / X)
$$




\subsection{Fake supersymmetric solutions}

In [15], Meessen and Palomo-Lozano presented a general method to obtain fake supersymmetric solutions to Wick-rotated $N=2, d=4$ gauged supergravity coupled to nonabelian vector multiplets. We will restrict ourselves here to the case of just abelian multiplets, with no gaugings of the scalar manifold's isometries; we will also consider only the timelike case of [15], which means that we take the norm of $V$ defined in (2.10) to be positive.

With these restrictions, the fake supersymmetric solutions always assume the form

$$
\begin{aligned}
d s^{2} & =2|X|^{2}(d \tau+\omega)^{2}-\frac{1}{2|X|^{2}} h_{m n} d y^{m} d y^{n}, \\
A^{\Lambda} & =-\frac{1}{2} \mathcal{R}^{\Lambda} V+\tilde{A}_{m}^{\Lambda} d y{ }^{m} \\
Z^{\Lambda} & =\frac{\mathcal{L}^{\Lambda}}{\mathcal{L}^{0}}=\frac{\mathcal{R}^{\Lambda}+i \mathcal{I}^{\Lambda}}{\mathcal{R}^{0}+i \mathcal{I}^{0}}
\end{aligned}
$$

where $V=2 \sqrt{2}|X|^{2}(d \tau+\omega), \omega=\omega_{m} d y^{m}$ is a 1 -form which can in general depend on $\tau$, and $h$ is the metric on a three-dimensional Gauduchon-Tod [18] base space. In particular there must exist a dreibein $W^{x}$ for $h$ satisfying

$$
d W^{x}=g C_{\Lambda} \tilde{A}^{\Lambda} \wedge W^{x}+\frac{g}{2 \sqrt{2}} C_{\Lambda} \mathcal{I}^{\Lambda} \varepsilon^{x y z} W^{y} \wedge W^{z}
$$

Furthermore the following equations must hold:

$$
\begin{aligned}
& \omega=g C_{\Lambda} \tilde{A}^{\Lambda} \tau+\tilde{\omega}, \\
& \tilde{F}_{x y}^{\Lambda}=-\frac{1}{\sqrt{2}} \varepsilon^{x y z} \tilde{\mathbb{D}}_{z} \mathcal{I}^{\Lambda}, \\
& \partial_{\tau} \mathcal{I}^{\Lambda}=0, \quad \partial_{\tau} \mathcal{I}_{\Lambda}=-\frac{g}{2 \sqrt{2}} C_{\Lambda}, \\
& \tilde{\mathbb{D}}_{x}^{2} \tilde{\mathcal{I}}_{\Lambda}-\left(\tilde{\mathbb{D}}_{x} \tilde{\omega}_{x}\right) \partial_{\tau} \mathcal{I}_{\Lambda}=0, \\
& \tilde{\mathbb{D}} \tilde{\omega}=\frac{1}{2} \varepsilon^{x y z}\left\langle\tilde{\mathcal{I}} \mid \partial_{x} \tilde{\mathcal{I}}-\tilde{\omega}_{x} \partial_{\tau} \mathcal{I}\right\rangle W^{y} \wedge W^{z},
\end{aligned}
$$

with

$$
\begin{aligned}
\tilde{F}^{\Lambda} & \equiv d \tilde{A}^{\Lambda},\left.\quad \tilde{\omega} \equiv \omega\right|_{\tau=0},\left.\quad \tilde{\mathcal{I}} \equiv \mathcal{I}\right|_{\tau=0} \\
\tilde{\mathbb{D}}_{m} \mathcal{I} & \equiv \partial_{m} \mathcal{I}+g C_{\Lambda} \tilde{A}_{m}^{\Lambda} \mathcal{I}, \quad \tilde{\mathbb{D}}_{x} \mathcal{I} \equiv W_{x}^{m} \tilde{\mathbb{D}}_{m} \mathcal{I}
\end{aligned}
$$

To obtain a specific solution we will then have to take the following steps: 
1. Choose the number of vector multiplets, the real constants $C_{\Lambda}$ and the special geometric manifold, e.g. by specifying a prepotential; this completely determines the bosonic action and permits to derive the dependence of the $\mathcal{R}$ 's from the $\mathcal{I}$ 's, the so-called stabilization equations.

2. Choose a three-dimensional Gauduchon-Tod base space, that is, choose a solution $\left(W^{x}, C_{\Lambda} \tilde{A}^{\Lambda}, C_{\Lambda} \mathcal{I}^{\Lambda}\right)$ of equation (2.15).

3. Determine the $\mathcal{I}^{\Lambda}$ 's and the $\tilde{A}^{\Lambda}$ 's that respect the choices of points 1 and 2 and at the same time satisfy equation (2.17).

4. Determine the $\mathcal{I}_{\Lambda}$ 's and $\tilde{\omega}$ from (2.18) and the coupled equations (2.19) and (2.20).

5. Solve the stabilization equations to find the $\mathcal{R}$ 's and finally write down the metric and the fields of the solution using (2.16) and $1 /|X|^{2}=2\langle\mathcal{R} \mid \mathcal{I}\rangle$.

In the next sections, we will use this procedure to find some solutions to theories with one vector multiplet, so that there will be only one physical scalar $Z^{1} \equiv Z$. We shall also make the simplest possible choice for the Gauduchon-Tod base space, the flat space.

\section{The $\mathcal{F}(\chi)=-\frac{i}{4}\left(\chi^{0}\right)^{n}\left(\chi^{1}\right)^{2-n}$ model}

Given this prepotential with $n \neq 0,2$, from (2.4) we can derive the Kähler potential

$$
e^{-\mathcal{K}}=\frac{n}{4} Z^{2-n}+\frac{2-n}{4} \bar{Z} Z^{1-n}+\text { c.c. },
$$

where we took $\left|\chi^{0}\right|=1$.

If we consider the truncation $\mathfrak{I m}(Z)=0$, the Kähler metric becomes

$$
\mathcal{G}=\left.\partial_{Z} \partial_{\bar{Z}} \mathcal{K}\right|_{\mathfrak{I m}(Z)=0}=\frac{n(2-n)}{4} \mathfrak{R e}(Z)^{-2}=\frac{n_{0} n_{1}}{16} e^{-2 \phi}
$$

where we defined $n_{0} \equiv 2 n, n_{1} \equiv 2(2-n)=4-n_{0}, \phi \equiv \log \mathfrak{R e}(Z)$.

From equation (2.6) we obtain then

$$
\mathcal{N}=-\frac{i}{8}\left(\begin{array}{cc}
n_{0} e^{\frac{n_{1}}{2} \phi} & 0 \\
0 & n_{1} e^{-\frac{n_{0}}{2} \phi}
\end{array}\right)
$$

and for the scalar potential (2.8) we get

$$
V=\frac{1}{2}\left[\frac{n_{0}\left(n_{0}-1\right)}{t_{0}^{2}} e^{-\frac{n_{1}}{2} \phi}+2 \frac{n_{0} n_{1}}{t_{0} t_{1}} e^{\frac{n_{0}-n_{1}}{4} \phi}+\frac{n_{1}\left(n_{1}-1\right)}{t_{1}^{2}} e^{\frac{n_{0}}{2} \phi}\right],
$$


with the definition $t_{\Lambda} \equiv-\frac{n_{\Lambda}}{2 g C_{\Lambda}}$. If one wishes to have a non-zero potential in the particular cases $n_{0}=1$ and $n_{0}=3$ one has to require respectively $C_{1} \neq 0$ and $C_{0} \neq 0$.

Plugging these expressions into (2.7) leads to the bosonic Lagrangian

$$
\begin{aligned}
e^{-1} \mathcal{L}= & R+\frac{n_{0} n_{1}}{8} \partial_{\mu} \phi \partial^{\mu} \phi-\frac{n_{0}}{4} e^{\frac{n_{1}}{2} \phi} F_{\mu \nu}^{0} F^{0 \mu \nu}-\frac{n_{1}}{4} e^{-\frac{n_{0}}{2} \phi} F_{\mu \nu}^{1} F^{1 \mu \nu} \\
& -\frac{1}{2}\left[\frac{n_{0}\left(n_{0}-1\right)}{t_{0}^{2}} e^{-\frac{n_{1}}{2} \phi}+2 \frac{n_{0} n_{1}}{t_{0} t_{1}} e^{\frac{n_{0}-n_{1}}{4} \phi}+\frac{n_{1}\left(n_{1}-1\right)}{t_{1}^{2}} e^{\frac{n_{0}}{2} \phi}\right] .
\end{aligned}
$$

We see that in order to avoid ghost fields in the Lagrangian one has to impose $0<n_{0}<$ 4 , corresponding to $0<n<2$ in the prepotential. One can check that for $t_{1} \rightarrow \infty$ (i.e., $\left.C_{1}=0\right)$, (3.5) reduces to the Lagrangian used in [17], if we identify $n_{0}=n_{T}, n_{1}=n_{S}$.

(2.11), together with $\mathfrak{I m}(Z)=0$, leads to

$$
\begin{array}{cc}
\mathcal{I}^{1}=e^{\phi} \mathcal{I}^{0}, & \mathcal{I}_{0}=\frac{n_{0}}{n_{1}} e^{\phi} \mathcal{I}_{1}, \\
\mathcal{R}^{0}=-\frac{8}{n_{1}} e^{\frac{n_{0}-n_{1}}{4} \phi} \mathcal{I}_{1}, & \mathcal{R}^{1}=-\frac{8}{n_{1}} e^{\frac{n_{0}}{2} \phi} \mathcal{I}_{1}, \\
\mathcal{R}_{0}=\frac{n_{0}}{8} e^{\frac{n_{1}}{2} \phi} \mathcal{I}^{0}, & \mathcal{R}_{1}=\frac{n_{1}}{8} e^{\frac{n_{1}-n_{0}}{4}} \mathcal{I}^{0},
\end{array}
$$

as well as

$$
\frac{1}{2|X|^{2}}=\langle\mathcal{R} \mid \mathcal{I}\rangle=\frac{1}{2} e^{\frac{n_{1}}{2} \phi}\left(\mathcal{I}^{0}\right)^{2}+\frac{32}{n_{1}^{2}} e^{\frac{n_{0}}{2} \phi}\left(\mathcal{I}_{1}\right)^{2} .
$$

Notice that since both $\mathcal{I}^{0}$ and $\mathcal{I}^{1}$ must be independent of $\tau$, either $\mathcal{I}^{0}=0$ or $\phi$ is also independent of $\tau$. In this second case using (2.18) we see that $C_{0}=0 \Leftrightarrow C_{1}=0$, so that if we require a non vanishing scalar potential we must impose $C_{0}, C_{1} \neq 0$; we also find that $e^{\phi}=\frac{n_{1}}{n_{0}} \frac{C_{0}}{C_{1}}=\frac{t_{1}}{t_{0}}$.

\subsection{Construction of the solution}

The simplest solution of eq. (2.15) is the flat three-dimensional space, with

$$
W_{m}^{x}=\delta_{m}^{x}, \quad C_{\Lambda} \tilde{A}^{\Lambda}=C_{\Lambda} \mathcal{I}^{\Lambda}=0
$$

With this choice for the base space we don't need to distinguish between $x, y, z \ldots$ and lower $m, n, p, \ldots$ indices.

If we require $V \neq 0, C_{\Lambda} \mathcal{I}^{\Lambda}=0$ together with (3.6) implies either

$$
\mathcal{I}^{0}=0 \quad \Rightarrow \quad \mathcal{I}^{1}=\mathcal{R}_{0}=\mathcal{R}_{1}=0,
$$

or a constant $\phi$ with

$$
e^{\phi}=-\frac{C_{0}}{C_{1}}
$$


and $C_{0}, C_{1} \neq 0$; but if $\phi$ is constant we should also have $e^{\phi}=\frac{n_{1}}{n_{0}} \frac{C_{0}}{C_{1}}$, so this choice is clearly inconsistent. The only consistent possibility is then $\mathcal{I}^{0}=0$. Using equation (2.17) this immediately implies

$$
\tilde{F}^{0}=\tilde{F}^{1}=0
$$

Because of (3.9) and $C_{\Lambda} \tilde{A}^{\Lambda}=0$, eq. (2.20) implies $d \tilde{\omega}=0$, and thus locally $\tilde{\omega}=d f$, where $f$ is a generic function of the spatial coordinates.

Equation (2.19) then becomes

$$
\left\{\begin{array} { l } 
{ \partial _ { p } \partial _ { p } ( \tilde { \mathcal { I } } _ { 0 } + \frac { g C _ { 0 } } { 2 \sqrt { 2 } } f ) = 0 , } \\
{ \partial _ { p } \partial _ { p } ( \tilde { \mathcal { I } } _ { 1 } + \frac { g C _ { 1 } } { 2 \sqrt { 2 } } f ) = 0 , }
\end{array} \quad \Rightarrow \quad \left\{\begin{array}{l}
\partial_{p} \partial_{p}\left(e^{\tilde{\phi}} \tilde{\mathcal{I}}_{1}-\frac{n_{1}}{4 \sqrt{2}} \frac{f}{t_{0}}\right)=0 \\
\partial_{p} \partial_{p}\left(\tilde{\mathcal{I}}_{1}-\frac{n_{1}}{4 \sqrt{2}} \frac{f}{t_{1}}\right)=0
\end{array}\right.\right.
$$

with $\left.\tilde{\phi} \equiv \phi\right|_{\tau=0}$. This can be solved by introducing two generic harmonic functions of the spatial coordinates $\mathcal{H}_{0}, \mathcal{H}_{1}$ as

$$
\tilde{\mathcal{I}}_{1}=\frac{n_{1}}{4 \sqrt{2}}\left(f / t_{1}+\mathcal{H}_{1}\right), \quad e^{\tilde{\phi}}=\frac{f / t_{0}+\mathcal{H}_{0}}{f / t_{1}+\mathcal{H}_{1}} .
$$

At this point, using (2.18) and $\mathcal{I}_{0}=e^{\phi} \mathcal{I}_{1}$ we obtain

$$
\begin{gathered}
\mathcal{I}_{1}=\frac{n_{1}}{4 \sqrt{2}}\left(\frac{\tau+f}{t_{1}}+\mathcal{H}_{1}\right), \quad \mathcal{I}_{0}=\frac{n_{0}}{4 \sqrt{2}}\left(\frac{\tau+f}{t_{0}}+\mathcal{H}_{0}\right), \\
e^{\phi}=\frac{(\tau+f) / t_{0}+\mathcal{H}_{0}}{(\tau+f) / t_{1}+\mathcal{H}_{1}}
\end{gathered}
$$

and from (3.7) one gets

$$
\frac{1}{2|X|^{2}}=\left(\frac{\tau+f}{t_{0}}+\mathcal{H}_{0}\right)^{\frac{n_{0}}{2}}\left(\frac{\tau+f}{t_{1}}+\mathcal{H}_{1}\right)^{\frac{n_{1}}{2}}
$$

We have now all the elements needed to write down the complete solution in terms of the two generic harmonic functions $\mathcal{H}_{0}$ and $\mathcal{H}_{1}$. Since $f$ appears everywhere as a shift in the time coordinate $\tau$ we can set it equal to zero with the coordinate change $t=\tau+f$ to obtain

$$
\begin{gathered}
d s^{2}=\mathcal{U}^{-2} d t^{2}-\mathcal{U}^{2} d \vec{y}^{2} \\
A^{\Lambda}=\left(\frac{t}{t_{\Lambda}}+\mathcal{H}_{\Lambda}\right)^{-1} d t, \quad \phi=\ln \left(\frac{t / t_{0}+\mathcal{H}_{0}}{t / t_{1}+\mathcal{H}_{1}}\right)
\end{gathered}
$$

with

$$
\mathcal{U} \equiv\left(\frac{t}{t_{0}}+\mathcal{H}_{0}\right)^{\frac{n_{0}}{4}}\left(\frac{t}{t_{1}}+\mathcal{H}_{1}\right)^{\frac{n_{1}}{4}}
$$

Here one clearly recognizes the substitution principle originally put forward by Behrndt and Cvetič in [19], which amounts to adding a linear time dependence to the harmonic functions in a supersymmetric solution of $N=2, d=4$ supergravity. 


\subsection{Physical discussion}

As a first remark if we set $C_{1}=0$, corresponding to $t_{1} \rightarrow \infty$, and make the choice of harmonic functions

$$
\mathcal{H}_{0}=\sum_{i=1}^{N} \frac{Q_{0}^{(i)}}{\left|\vec{y}-\overrightarrow{y_{i}}\right|}, \quad \mathcal{H}_{1}=1+\sum_{i=1}^{N} \frac{Q_{1}^{(i)}}{\left|\vec{y}-\vec{y}_{i}\right|},
$$

we recover precisely the solution presented in [17]. The same is true if we set $C_{0}=0$, change the sign of the scalar field and exchange everywhere 0 and 1 indices. This solution represents a system of multiple maximally charged black holes in a universe expanding with arbitrary equation of state $P=w \rho$, with $w=\frac{8-5 n_{0}}{3 n_{0}}$ so that $-1 \leq w \leq 1$ for $1 \leq n_{0} \leq 4$ (for $n_{0}<1$ the scalar potential is unbounded from below). Note that one can have $w<-1$ by allowing $n_{0}<0$ or $n_{0}>4$, but then of course the action (3.5) contains ghosts. In this case, we would have black holes embedded in an expanding universe filled with phantom energy. In the limit $n_{0}=4$ one obtains the Kastor-Traschen solution [6], describing multiple black holes in a de Sitter background, while for $n_{0}=0$ the scalar potential is zero and the solution is the Majumdar-Papapetrou spacetime, describing multiple extremal Reissner-Nordström black holes in an asymptotically flat background. Notice that we can also recover the Kastor-Traschen solution keeping both $t_{0}$ and $t_{1}$ finite and taking $t_{0} \mathcal{H}_{0}=t_{1} \mathcal{H}_{1}$.

Retaining both $C_{0}$ and $C_{1}$ the scalar potential has critical points; the derivative of the scalar potential can be written as

$$
V^{\prime}[\phi]=\frac{n_{0} n_{1}}{4 t_{1}^{2}} e^{-\frac{n_{1}}{4} \phi}\left[\frac{t_{1}}{t_{0}}-e^{\phi}\right]\left[\frac{t_{1}}{t_{0}}\left(1-n_{0}\right)+\left(1-n_{1}\right) e^{\phi}\right] .
$$

We can see that if we take $t_{0} t_{1}>0$ there is, for every value of $0<n_{0}<4$, a minimum in $e^{\phi}=\frac{t_{1}}{t_{0}}, V_{\min }=6 t_{1}^{-n_{1} / 2} t_{0}^{-n_{0} / 2}$. For $0<n_{0}<1$ or $3<n_{0}<4$ there is also a maximum in $e^{\phi}=-\frac{1-n_{0}}{1-n_{1}} \frac{t_{1}}{t_{0}}, V_{\max }=2\left(\frac{n_{0}-1}{1-n_{1}}\right)^{\frac{n_{0}-n_{1}}{4}} t_{1}^{-n_{1} / 2} t_{0}^{-n_{0} / 2}$; however for these values of $n_{0}$ the potential is not bounded from below. For $1<n_{0}<3$ the potential is bounded and the minimum is global.

If on the other hand we take $t_{0} t_{1}<0$, there is only a negative minimum in $e^{\phi}=$ $-\frac{1-n_{0}}{1-n_{1}} \frac{t_{1}}{t_{0}}$ if $1<n_{0}<3, V_{\min }=-2\left(\frac{1-n_{0}}{1-n_{1}}\right)^{\frac{n_{0}-n_{1}}{4}}\left|t_{1}\right|^{-n_{1} / 2}\left|t_{0}\right|^{-n_{0} / 2}$, while there are no critical points for $0<n_{0}<1$ or $3<n_{0}<4$.

For $t_{0} t_{1}>0$ and assuming that the harmonic functions have a well-defined limit for $|\vec{y}| \rightarrow \infty$, one can study the asymptotic behaviour of the metric; swapping the coordinate $t$ for $\tilde{t}$ defined by

$$
\frac{d \tilde{t}}{d t}=\left(\frac{t}{t_{0}}+k_{0}\right)^{-\frac{n_{0}}{4}}\left(\frac{t}{t_{1}}+k_{1}\right)^{-\frac{n_{1}}{4}}, \quad k_{i} \equiv \lim _{|\vec{y}| \rightarrow \infty} \mathcal{H}_{i}
$$


the metric asymptotically assumes a Friedmann-Lemaître-Robertson-Walker form,

$$
d s^{2}=d \tilde{t}^{2}-a^{2}(\tilde{t}) d \vec{y}^{2}, \quad a(\tilde{t})=\frac{d t}{d \tilde{t}} .
$$

The explicit form of $a(\tilde{t})$ is complicated; however it is possible to obtain the timedependence of the density and pressure,

$$
\begin{aligned}
\rho(\tilde{t}) & =\frac{3}{128 \pi} \frac{n_{0}^{2}}{t_{0}^{2}} \frac{1}{R(\tilde{t})^{\frac{n_{0}}{2}}}\left(R(\tilde{t})+\frac{n_{1}}{n_{0}} \frac{t_{0}}{t_{1}}\right)^{2}, \\
P(\tilde{t}) & =-\frac{5}{128 \pi} \frac{n_{0}^{2}}{t_{0}^{2}} \frac{1}{R(\tilde{t})^{\frac{n_{0}}{2}}}\left[\left(R(\tilde{t})+\frac{n_{1}}{n_{0}} \frac{t_{0}}{t_{1}}\right)^{2}-\frac{8}{5 n_{0}}\left(R^{2}(\tilde{t})+\frac{n_{1}}{n_{0}} \frac{t_{0}^{2}}{t_{1}^{2}}\right)\right],
\end{aligned}
$$

where

$$
R(\tilde{t}) \equiv \frac{t(\tilde{t}) / t_{1}+k_{1}}{t(\tilde{t}) / t_{0}+k_{0}}
$$

so that

$$
\frac{P(\tilde{t})}{\rho(\tilde{t})}=w(\tilde{t})=-\frac{5}{3}\left[1-\frac{8}{5 n_{0}} \frac{R^{2}(\tilde{t})+\frac{n_{1}}{n_{0}} \frac{t_{0}^{2}}{t_{1}^{2}}}{\left(R(\tilde{t})+\frac{n_{1}}{n_{0}} \frac{t_{0}}{t_{1}}\right)^{2}}\right],
$$

that gives the correct value of [17] in the limits $t_{0} \rightarrow \infty$ or $t_{1} \rightarrow \infty$.

If both $t_{0}$ and $t_{1}$ are finite, $w$ is time-independent only if $t_{0} k_{0}=t_{1} k_{1}$, which is equivalent to consider $k_{0}=k_{1}=0$, since we are free to set $k_{0}=0$ without loss of generality by shifting $t$. In this case $a(\tilde{t})=e^{\tilde{t} / \tilde{t}_{0}}$, with $\tilde{t}_{0}=t_{0}^{n_{0} / 4} t_{1}^{n_{1} / 4}, w=-1$ and the spacetime is asymptotically de Sitter independently of the value of $n_{0}$, while the scalar field tends to the critical value $e^{\phi}=t_{1} / t_{0}$.

Note that in the case $t_{0} t_{1}>0$, the solution (3.16) tends to de Sitter for $|\vec{y}| \rightarrow \infty$ and arbitrary $k_{\Lambda}$ either for $t \rightarrow \infty$ or $t \rightarrow-\infty$ (for positive or negative $t_{\Lambda}$ respectively).

Since we are interested in black hole systems, we consider harmonic functions of the form

$$
H_{\Lambda}(t, \vec{y}) \equiv \frac{t}{t_{\Lambda}}+\mathcal{H}_{\Lambda}=\frac{t}{t_{\Lambda}}+k_{\Lambda}+\sum_{i=1}^{N} \frac{Q_{\Lambda}^{(i)}}{\left|\vec{y}-\vec{y}_{i}\right|}
$$

and take $k_{0}=0$ since it can be eliminated by shifting $t$. Notice that while we could take some of the charges to be zero, this would lead to a divergent scalar field in the limit $\left|\vec{y}-\vec{y}_{i}\right| \rightarrow 0$. 
The scalar curvature of $(3.16)$ reads

$$
\begin{aligned}
R= & \frac{3}{8} \frac{n_{1}\left(3 n_{1}-4\right) t_{0}^{2} H_{0}^{2}+6 n_{0} n_{1} t_{0} t_{1} H_{0} H_{1}+n_{0}\left(3 n_{0}-4\right) t_{1}^{2} H_{1}^{2}}{t_{0}^{2} t_{1}^{2} H_{0}^{\frac{n_{1}}{2}} H_{1}^{\frac{n_{0}}{2}}} \\
& +\frac{n_{0} n_{1}}{8 H_{0}^{\frac{n_{0}}{2}} H_{1}^{\frac{n_{1}}{2}}}\left(\partial_{p} \ln \frac{H_{0}}{H_{1}}\right)^{2},
\end{aligned}
$$

which is singular for $H_{0}=0$ or $H_{1}=0$.

We can also consider the limit $\left|\vec{y}-\vec{y}_{i}\right| \equiv r_{i} \rightarrow 0$ for some $i$; then the time dependence drops out and the metric reduces to $\mathrm{AdS}_{2} \times \mathrm{S}^{2}$,

$$
d s_{r_{i} \rightarrow 0}^{2}=\frac{r_{i}^{2}}{l_{i}^{2}} d t^{2}-\frac{l_{i}^{2}}{r_{i}^{2}} d r_{i}^{2}-l_{i}^{2} d \Omega_{2}^{2},
$$

with $l_{i} \equiv\left(Q_{0}^{(i)}\right)^{n_{0} / 4}\left(Q_{1}^{(i)}\right)^{n_{1} / 4}$. As we shall see later, (3.28) does actually not describe the geometry near the event horizon of our time-dependent solution.

We turn now to study in more detail the system with a single black hole. Since in this case there is spherical symmetry, we will work in spherical coordinates,

$$
H_{0}(t, r)=\frac{t}{t_{0}}+\frac{Q_{0}}{r}, \quad H_{1}(t, r)=\frac{t}{t_{1}}+k_{1}+\frac{Q_{1}}{r} .
$$

If $Q_{0}, Q_{1} \neq 0$ we will assume in the following, without loss of generality, $\left|Q_{1} t_{1}\right| \geq\left|Q_{0} t_{0}\right|$.
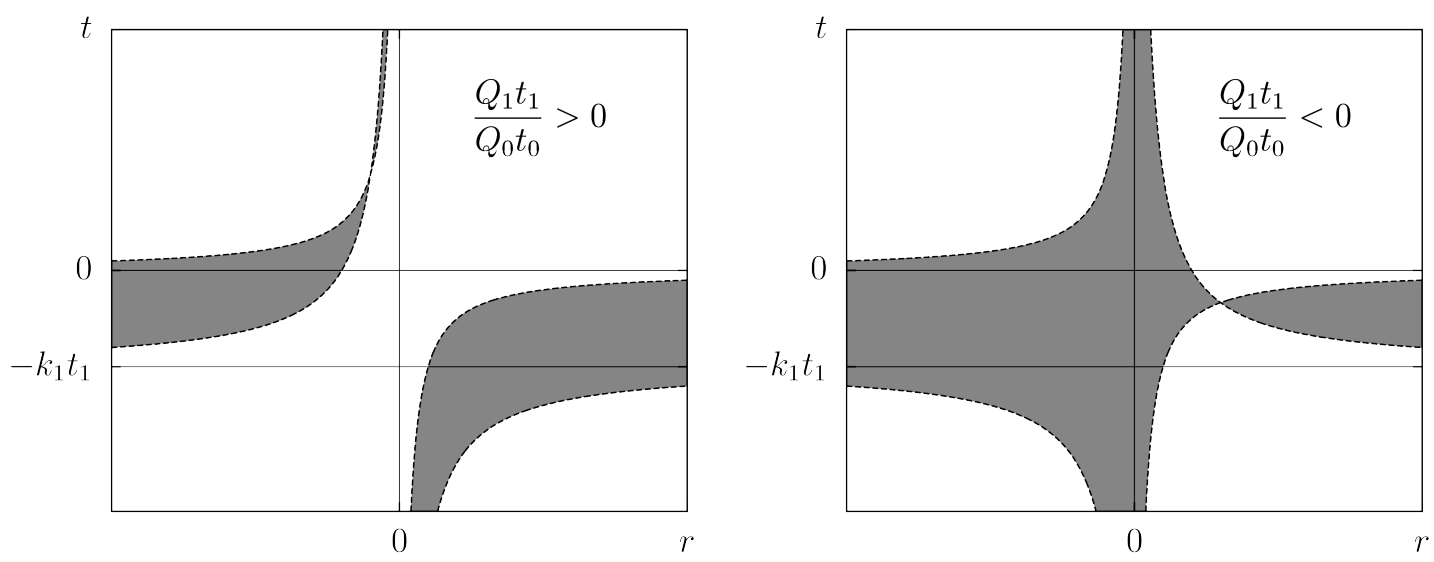

Figure 1. Allowed coordinate ranges in the $r-t$ plane. The dashed curves denote the curvature singularities, the allowed range is the white area for $t_{0} t_{1}>0$ or the grey area for $t_{0} t_{1}<0$. We assume here $k_{1} t_{1}$ and $Q_{0} t_{0}$ positive; the other cases can be obtained by reflection or rotation. 
Since $r=0$ is not a curvature singularity unless one of the charges is zero, the spacetime can be extended to $r<0$. The singularities are represented in the $r$ - $t$ plane by two hyperbolae having the asymptotes $r=0$ and respectively $t=0$ or $t=-k_{1} t_{1}$; if $k_{1} \neq 0$ they intersect unless $Q_{0} t_{0}=Q_{1} t_{1}$. To ensure the regularity of the solution we must require $H_{0} H_{1}>0$; this corresponds to the area external to the singularities in the $r$ - $t$ plane for $t_{0} t_{1}>0$ or to the area between them if $t_{0} t_{1}<0$ (see figure 1 ).

The present spacetime satisfies the weak energy condition; to see this, compute the energy-momentum tensor components $T_{a b}$ for an observer with orthonormal frame

$$
e^{0}=\mathcal{U}^{-1} d t, \quad e^{1}=\mathcal{U} d r, \quad e^{2}=\mathcal{U} r d \theta, \quad e^{3}=\mathcal{U} r \sin \theta d \varphi
$$

One obtains

$$
\begin{aligned}
& \rho^{\phi}=\mathcal{F}_{1}+\mathcal{F}_{2}+\mathcal{F}_{3}, \quad P_{r}^{\phi}=\mathcal{F}_{1}+\mathcal{F}_{2}-\mathcal{F}_{3}, \quad P_{\Omega}^{\phi}=\mathcal{F}_{1}-\mathcal{F}_{2}-\mathcal{F}_{3}, \\
& \rho^{\mathrm{em}}=-P_{r}^{\mathrm{em}}=P_{\Omega}^{\mathrm{em}}=\mathcal{F}_{4}, \quad T_{01}^{\phi}=-\mathcal{F}_{5},
\end{aligned}
$$

where $\rho=T_{00}, P_{r}=T_{11}, P_{\Omega}=T_{22}=T_{33}$, the other off-diagonal components are zero, and

$$
\begin{gathered}
\mathcal{F}_{1}=\mathcal{U}^{2} \frac{n_{0} n_{1}}{16}\left(\frac{1}{t_{0} H_{0}}-\frac{1}{t_{1} H_{1}}\right)^{2}, \quad \mathcal{F}_{2}=\mathcal{U}^{-2} \frac{n_{0} n_{1}}{16 r^{4}}\left(\frac{Q_{0}}{H_{0}}-\frac{Q_{1}}{H_{1}}\right)^{2} \\
\mathcal{F}_{3}=\frac{\mathcal{U}^{2}}{4}\left[\left(\frac{n_{0}}{t_{0} H_{0}}-\frac{n_{1}}{t_{1} H_{1}}\right)^{2}-\frac{n_{0}}{t_{0}^{2} H_{0}^{2}}-\frac{n_{1}}{t_{1}^{2} H_{1}^{2}}\right], \quad \mathcal{F}_{4}=\frac{\mathcal{U}^{-2}}{4 r^{4}}\left(\frac{n_{0} Q_{0}^{2}}{H_{0}^{2}}+\frac{n_{1} Q_{1}^{2}}{H_{1}^{2}}\right), \\
\mathcal{F}_{5}=\frac{n_{0} n_{1}}{8 r^{2}}\left(\frac{1}{t_{0} H_{0}}-\frac{1}{t_{1} H_{1}}\right)\left(\frac{Q_{0}}{H_{0}}-\frac{Q_{1}}{H_{1}}\right) .
\end{gathered}
$$

Since $\mathcal{F}_{1}, \mathcal{F}_{2}, \mathcal{F}_{4}$ and $\mathcal{F}_{1}+\mathcal{F}_{3}=\mathcal{U}^{2} \frac{3}{16}\left(\frac{n_{0}}{t_{0} H_{0}}+\frac{n_{1}}{t_{1} H_{1}}\right)^{2}$ are positive definite, the energy densities $\rho^{\phi}$ and $\rho^{\text {em }}$ are positive. Notice also that $\mathcal{F}_{4}-\mathcal{F}_{2}=\mathcal{U}^{-2}\left(\frac{n_{0} Q_{0}}{H_{0}}+\frac{n_{1} Q_{1}}{H_{1}}\right)^{2} /\left(16 r^{4}\right)$ is positive definite and that $\mathcal{F}_{5}^{2}=4 \mathcal{F}_{1} \mathcal{F}_{2}$.

$T_{b}^{a}$ can always be diagonalized by changing to a different orthonormal basis. Its eigenvalues are

$$
\begin{aligned}
\hat{\rho} & =\frac{1}{2}\left(\rho-P_{r}+\sqrt{\left(\rho+P_{r}\right)^{2}-4 T_{01}^{2}}\right), \\
-\hat{P}_{r} & =-\frac{1}{2}\left(\rho-P_{r}+\sqrt{\left(\rho+P_{r}\right)^{2}-4 T_{01}^{2}}\right), \\
-\hat{P}_{\Omega} & =-P_{\Omega} .
\end{aligned}
$$

In terms of these the weak energy condition can be stated as

$$
\hat{\rho} \geq 0, \quad \hat{\rho}+\hat{P}_{r} \geq 0, \quad \hat{\rho}+\hat{P}_{\Omega} \geq 0 .
$$


We have

$$
\begin{aligned}
& \hat{\rho}=\mathcal{F}_{3}+\mathcal{F}_{4}+\left|\mathcal{F}_{1}-\mathcal{F}_{2}\right| \geq\left(\mathcal{F}_{1}+\mathcal{F}_{3}\right)+\left(\mathcal{F}_{4}-\mathcal{F}_{2}\right) \geq 0 \\
& \hat{\rho}+\hat{P}_{r}=2\left|\mathcal{F}_{1}-\mathcal{F}_{2}\right| \geq 0 \\
& \hat{\rho}+\hat{P}_{\Omega}=\mathcal{F}_{1}+\mathcal{F}_{4}+\left(\mathcal{F}_{4}-\mathcal{F}_{2}\right)+\left|\mathcal{F}_{1}-\mathcal{F}_{2}\right| \geq 0
\end{aligned}
$$

and thus (3.34) holds. Whether the strong and dominant energy conditions are satisfied depends on the values of the parameters; it has been shown in particular that the Gibbons-Maeda solution $\left(t_{1} \rightarrow \infty\right)$ satisfies the strong energy condition if and only if the asymptotic cosmological background does [11], and that the Maeda-Ohta-Uzawa solution $\left(t_{1} \rightarrow \infty, n_{0}=1\right)$ satisfies the dominant energy condition [10].

The spherical symmetry allows us to covariantly define the circumference radius $R=|r| \mathcal{U}=|r| H_{0}^{n_{0} / 4} H_{1}^{n_{1} / 4}$; it is immediate to see that this radius vanishes on the singularities. In a spherically symmetric spacetime it is also possible to compute the Misner-Sharp quasilocal energy [20], that can be interpreted as the energy inside a closed surface of radius $R$,

$$
m=4 \pi R\left(1+\nabla_{\mu} R \nabla^{\mu} R\right)
$$

where

$$
\nabla_{\mu} R \nabla^{\mu} R=-\frac{1}{16}\left[\left(\frac{t n_{0}}{t_{0} H_{0}}+\frac{\left(t+k_{1} t_{1}\right) n_{1}}{t_{1} H_{1}}\right)^{2}-r^{2} H_{0}^{n_{0}} H_{1}^{n_{1}}\left(\frac{n_{0}}{t_{0} H_{0}}+\frac{n_{1}}{t_{1} H_{1}}\right)^{2}\right] .
$$

Following [10, 11] we can look for trapping horizons [21]. Introducing the NewmanPenrose null tetrads

$$
\begin{aligned}
l & =\frac{1}{\sqrt{2}}\left(\mathcal{U}^{-1} d t-\mathcal{U} d r\right), \\
n & =\frac{1}{\sqrt{2}}\left(\mathcal{U}^{-1} d t+\mathcal{U} d r\right), \\
m & =\mathcal{U} \frac{r}{\sqrt{2}}(d \theta+i \sin \theta d \varphi),
\end{aligned}
$$

and the complex conjugate $\bar{m}$, satisfying $l^{\mu} n_{\mu}=1=-m^{\mu} \bar{m}_{\mu}$, the expansions of the outgoing and ingoing radial null geodesics are defined by

$$
\theta_{+} \equiv-2 m^{(\mu} \bar{m}^{\nu)} \nabla_{\mu} l_{\nu}, \quad \theta_{-} \equiv-2 m^{(\mu} \bar{m}^{\nu)} \nabla_{\mu} n_{\nu}
$$

which evaluated explicitly are

$$
\theta_{ \pm}=\frac{1}{2 \sqrt{2} r \mathcal{U}}\left[r \mathcal{U}^{2}\left(\frac{n_{0}}{t_{0} H_{0}}+\frac{n_{1}}{t_{1} H_{1}}\right) \pm\left(\frac{t n_{0}}{t_{0} H_{0}}+\frac{\left(t+k_{1} t_{1}\right) n_{1}}{t_{1} H_{1}}\right)\right]
$$


While $\theta_{ \pm}$are not covariant quantities, their product is; comparing (3.39) and (3.42) it is straightforward to conclude that

$$
\theta_{+} \theta_{-}=\frac{2}{R^{2}} \nabla_{\mu} R \nabla^{\mu} R
$$

A metric sphere is said to be trapped or untrapped if $\theta_{+} \theta_{-}>0$ or $\theta_{+} \theta_{-}<0$ respectively, and to be marginal if $\theta_{+} \theta_{-}=0$. A trapping horizon is the closure of a hypersurface foliated by marginal surfaces, which means that it occurs when $\theta_{+} \theta_{-}=0$, or equivalently when $\nabla_{\mu} R$ becomes null.

It is possible to geometrically define on trapping horizons a local surface gravity $k_{l}$ and the associated Hawking temperature $T_{l}=\frac{k_{l}}{2 \pi}[22,23]$,

$$
\begin{gathered}
k_{l} \equiv-\left.\frac{1}{2} \tilde{\nabla}_{\mu} \tilde{\nabla}^{\mu} R\right|_{\mathrm{TH}}= \\
-\frac{1}{8 R}\left\{\left(\frac{t n_{0} t_{1} H_{1}+\left(t+k_{1} t_{1}\right) n_{1} t_{0} H_{0}}{n_{0} t_{1} H_{1}+n_{1} t_{0} H_{0}}\right)^{2}\left[\left(\frac{n_{0}}{t_{0} H_{0}}+\frac{n_{1}}{t_{1} H_{1}}\right)^{2}-\left(\frac{n_{0}}{t_{0}^{2} H_{0}^{2}}+\frac{n_{1}}{t_{1}^{2} H_{1}^{2}}\right)\right]\right. \\
\left.+\left(\frac{t^{2} n_{0}}{t_{0}^{2} H_{0}^{2}}+\frac{\left(t+k_{1} t_{1}\right)^{2} n_{1}}{t_{1}^{2} H_{1}^{2}}\right)-2\left(\frac{t n_{0}}{t_{0} H_{0}}+\frac{\left(t+k_{1} t_{1}\right) n_{1}}{t_{1} H_{1}}\right)\right\}
\end{gathered}
$$

where $\tilde{\nabla}$ is the covariant derivative associated with the two dimensional metric normal to the spheres of symmetry. This surface gravity satisfies on the trapping horizons an identity similar to the usual relation for stationary black holes,

$$
K^{\mu} \nabla_{[\nu} K_{\mu]}=k_{l} K_{\nu}
$$

where in place of a Killing vector we have the Kodama vector $K \equiv g^{-1}(* d R)$, with * evaluated with respect to the normal metric. It should be noted however that an observer whose worldline is an integral curve of $K$ does not measure the temperature $T_{l}$ near the trapping horizons; the observed temperature is, to first order, $T=T_{l} C^{-1 / 2}$, with redshift factor $C=\nabla_{\mu} R \nabla^{\mu} R$.

Now if we take $k_{1}=0$ or equivalently consider the limit $r \rightarrow 0, t \rightarrow \infty$ with $r t$ kept finite, (3.39) vanishes for $t^{2}=r^{2} H_{0}^{n_{0}} H_{1}^{n_{1}}$, i.e.,

$$
t^{2} r^{2}=\left(\frac{t r}{t_{0}}+Q_{0}\right)^{n_{0}}\left(\frac{t r}{t_{1}}+Q_{1}\right)^{n_{1}}
$$

or $n_{1} t_{0} H_{0}+n_{0} t_{1} H_{1}=0$ if $t_{0} t_{1}<0$. However the latter solution doesn't correspond to a change of sign in $\theta_{+} \theta_{-}$, so it doesn't identify a trapping horizon. Notice that the solutions of (3.46) have constant circumference radius $R$, and since the gradient of $R$ 
becomes null there, the trapped horizons are null surfaces in the limit $r \rightarrow 0, t \rightarrow \infty$ with $r$ fixed. In this limit the geometric surface gravity (3.44) simplifies to

$$
k_{l}=\frac{1}{8 R}\left(\frac{t n_{0}}{t_{0} H_{0}}+\frac{t n_{1}}{t_{1} H_{1}}\right)\left(2-\frac{t n_{0}}{t_{0} H_{0}}-\frac{t n_{1}}{t_{1} H_{1}}\right) .
$$

The identification of event horizons is a nontrivial task for dynamical black holes, since it requires the knowledge of the entire causal structure of the spacetime. Nevertheless, we can argue as in [10], and use the fact that the event horizon has to cover the trapped surfaces provided the outside region of a black hole behaves sufficiently well [24]. Since the spacetime (3.16) is indeed well-behaved for positive $r$ (as long as we are outside the forbidden regions in fig. 1), and the trapping horizons contain null surfaces (3.46) in the limit $r \rightarrow 0, t \rightarrow \infty$, we shall examine in the following if these null surfaces are possible candidates for the black hole event horizon. As we said, the limit $r \rightarrow 0, t \rightarrow \infty$ with $r t$ kept finite is equivalent to taking $k_{1}=0$. In this case, the metric is invariant under the transformation $t \rightarrow \alpha t, r \rightarrow r / \alpha$, and thus admits the Killing vector $\xi=t \partial_{t}-r \partial_{r}$, which is hypersurface orthogonal. Introducing the coordinates

$$
\begin{gathered}
T= \pm \log |t|+\int^{\mathcal{R}} \frac{g^{2}(\mathcal{R})}{\mathcal{R} f(\mathcal{R})} d \mathcal{R}, \quad \mathcal{R}=\frac{r t}{Q_{0} t_{0}} \\
f(\mathcal{R}) \equiv\left(Q_{0} t_{0}\right)^{2} \mathcal{R}^{2}-g^{2}(\mathcal{R}), \quad g(\mathcal{R}) \equiv Q_{0}^{2}(\mathcal{R}+1)^{\frac{n_{0}}{2}}\left(\frac{t_{0}}{t_{1}} \mathcal{R}+\frac{Q_{1}}{Q_{0}}\right)^{\frac{n_{1}}{2}}
\end{gathered}
$$

such that $\xi=\partial_{T}$, the metric can be written in static form as

$$
d s^{2}=\frac{f(\mathcal{R})}{g(\mathcal{R})} d T^{2}-\left(Q_{0} t_{0}\right)^{2} \frac{g(\mathcal{R})}{f(\mathcal{R})} d \mathcal{R}^{2}-g(\mathcal{R}) d \Omega^{2}
$$

From (3.50) it is clear that there are Killing horizons where $f(\mathcal{R})=0$, that is, in $(r, t)$ coordinates, $t^{2}=r^{2} H_{0}^{n_{0}} H_{1}^{n_{1}}$; thus the Killing horizons coincide with the trapping horizons (3.46). As the near-horizon geometry (3.50) enjoys the unexpected symmetry under translations of the time coordinate $T$ (which is not a symmetry of the original spacetime (3.16)), our solution (3.16) provides (like the ones in [9, 10]) a realization of asymptotic symmetry enhancement at the horizon of a dynamical black hole. The fact that the horizon does not grow, i.e., the ambient matter does not accrete onto the black hole, was conjectured in [13] to be related to fake supersymmetry.

Since the spacetime (3.50) is static, we can calculate the surface gravity on the horizons which is given by

$$
k^{2}=-\frac{1}{2} \nabla_{\mu} \xi_{\nu} \nabla^{\mu} \xi^{\nu}=\frac{1}{4}\left(\frac{n_{0} \mathcal{R}}{\mathcal{R}+1}+\frac{n_{1} \mathcal{R}}{\mathcal{R}+\frac{Q_{1} t_{1}}{Q_{0} t_{0}}}-2\right)^{2},
$$


that depends only on $\mathcal{R}$ (or equivalently on $r t$ ) and where $\mathcal{R}$ is one root of $f(\mathcal{R})=0$. Note that, contrary to the asymptotically flat case, there is no preferred normalization for the Killing vector $\xi$ here, and that the surface gravity is sensitive to this norm. Notice also that in general (3.51) is nonvanishing. A temperature different from zero would be in contradiction with supersymmetry, but not with fake supersymmetry: Following the explanation in [25], consider a black hole with temperature T. A spinor in the Euclidean section must then be antiperiodic under translation of the Euclidean time through a period $\beta=1 / T$. Supersymmetry implies the existence of a spinor field solving the Killing spinor equation, and this spinor must be periodic to give a regular solution. Both requirements are compatible only if the period is infinite, or equivalently when the temperature vanishes. Now, in fake supergravity, there are no fermions whose variation under a putative fake supersymmetry transformation is associated to the fake Killing spinor equation. The latter is just an auxiliary construction, which implies (under certain conditions) the second order field equations. Thus, the above contradiction for nonzero temperature does not arise.

Rewriting (3.47) in static coordinates,

$$
k_{l}=\frac{1}{8 \sqrt{\left|Q_{0} t_{0} \mathcal{R}\right|}}\left(\frac{n_{0} \mathcal{R}}{\mathcal{R}-1}+\frac{n_{1} \mathcal{R}}{\mathcal{R}-\frac{Q_{1} t_{1}}{Q_{0} t_{0}}}\right)\left(2-\frac{n_{0} \mathcal{R}}{\mathcal{R}-1}-\frac{n_{1} \mathcal{R}}{\mathcal{R}-\frac{Q_{1} t_{1}}{Q_{0} t_{0}}}\right),
$$

we see that it agrees with (3.51) up to a normalization factor constant over each Killing horizon. This is the same factor that ties the Kodama vector $K$ to the Killing vector $\xi$ on the horizons,

$$
\left.K\right|_{\mathrm{KH}}= \pm \frac{1}{4 \sqrt{\left|Q_{0} t_{0} \mathcal{R}\right|}}\left(\frac{n_{0} \mathcal{R}}{\mathcal{R}-1}+\frac{n_{1} \mathcal{R}}{\mathcal{R}-\frac{Q_{1} t_{1}}{Q_{0} t_{0}}}\right) \xi
$$

The horizon condition can be rewritten as

$$
|\mathcal{R}|=a|\mathcal{R}+1|^{\frac{n_{0}}{2}}|\mathcal{R}+b|^{\frac{n_{1}}{2}}, \quad a \equiv\left|\frac{Q_{0}}{t_{0}}\right|\left|\frac{t_{0}}{t_{1}}\right|^{\frac{n_{1}}{2}}, \quad b \equiv \frac{Q_{1} t_{1}}{Q_{0} t_{0}} .
$$

If $t_{0} t_{1}>0$ the accessible regions of spacetime are $\mathcal{R}>\max (-1,-b)$ and $\mathcal{R}<$ $\min (-1,-b)$.

We see that for $b>1$ there are always exactly two horizons for negative $\mathcal{R}$, one for $\mathcal{R}<-b$ and one for $-1<\mathcal{R}<0$; however only one of these is accessible since they are located in disconnected regions of the spacetime. For $a \geq 1 / 4$ one has $\mathcal{R} \leq$ $a(\mathcal{R}+1)^{2}<a(\mathcal{R}+1)^{n_{0} / 2}(\mathcal{R}+b)^{n_{1} / 2}$ for every positive $\mathcal{R}$ and consequently there are no other horizons. On the other hand if $a \leq 1 /(4 b)$ there is an interval for which $\mathcal{R} \geq a(\mathcal{R}+b)^{2}>a(\mathcal{R}+1)^{n_{0} / 2}(\mathcal{R}+b)^{n_{1} / 2}$ and there are thus two distinct horizons for 
positive $\mathcal{R}$. For intermediate values of $a$ there can be zero or two, possibly coincident, horizons for positive $\mathcal{R}$ depending on the value of the parameters. $b=1$ corresponds to the single-centered Kastor-Traschen solution, or Reissner-Nordström-de Sitter with mass equal to the charge, the extremal case corresponding to $a=1 / 4$. We can then identify the three horizons in the $\mathcal{R}>-1$ region as respectively inner and outer black hole horizons and cosmological horizon.

For $b \leq-1$ there is always one horizon in the region $\mathcal{R}>-b$ and at least one, at most three horizons for $\mathcal{R}<-1$. In this case $\mathcal{R}=0$ is not accessible.

For $b=0$, corresponding to a black hole charged under only one of the gauge fields, there is a solution in $\mathcal{R}=0$ which is not a horizon since it is coincident with a singularity; depending on the value of the parameters there can be zero, one or two horizons for $\mathcal{R}>0$. In the region $\mathcal{R}<-1$ there is always a single horizon.

If $t_{0} t_{1}<0$ the accessible region is given by the values of $\mathcal{R}$ between -1 and $-b$. For $b>1$ there can be zero, one or two, possibly coincident, horizons; for $b<-1$ there are always two horizons, one with negative and one with positive $\mathcal{R}$. For $b=0$ there is again a solution in $\mathcal{R}=0$ coincident with a singularity; depending on the value of the parameters there can be zero, one or two additional solutions corresponding to horizons.

With the choice of coordinates we made, the radial null geodesic equations simplify to

$$
\ddot{T}+2 \Gamma_{T \mathcal{R}}^{T} \dot{T} \dot{\mathcal{R}}=0, \quad \ddot{\mathcal{R}}=0,
$$

which means that $\mathcal{R}$ is an affine parameter for the radial null geodesics and consequently all horizons and singularities are reached within a finite value of the affine parameter. From the null condition $d \mathcal{R}= \pm f(\mathcal{R}) d T /\left(Q_{0} t_{0} g(\mathcal{R})\right)$ we obtain the expressions for the radial null geodesics in the near-horizon and near-singularity limits,

$$
\begin{array}{lll}
\mathcal{R} \sim \mathcal{R}_{\text {hor }}: & T= \pm \frac{1}{2 k} \log \left|\mathcal{R}-\mathcal{R}_{\text {hor }}\right|+c_{1}, \\
\mathcal{R} \sim-1: & T= \pm 2 \frac{Q_{0}}{t_{0}}\left(\frac{Q_{1}}{Q_{0}}-\frac{t_{0}}{t_{1}}\right)^{\frac{n_{1}}{2}} \frac{(\mathcal{R}+1)^{1+\frac{n_{0}}{2}}}{2+n_{0}}+c_{2}, \\
\mathcal{R} \sim-\frac{Q_{1} t_{1}}{Q_{0} t_{0}}: & T= \pm 2 \frac{Q_{1}}{t_{1}}\left(\frac{Q_{0}}{Q_{1}}-\frac{t_{1}}{t_{0}}\right)^{\frac{n_{0}}{2}} \frac{\left(\frac{Q_{0} t_{0}}{Q_{1} t_{1}} \mathcal{R}+1\right)^{1+\frac{n_{1}}{2}}}{2+n_{1}}+c_{3},
\end{array}
$$

where $c_{i}$ are constants and $k$ is the surface gravity (3.51).

\section{Alternative model}

In section 3 we considered the truncation $\mathfrak{I m}(Z)=0$; we could also have taken $\mathfrak{R e}(Z)=$ 0 , but this choice is not consistent for every value of $n$ with the prepotential we had 
there. Here we consider a slightly modified prepotential,

$$
\mathcal{F}(\chi)=\frac{i^{n-1}}{4(1-n)}\left(\chi^{0}\right)^{n}\left(\chi^{1}\right)^{2-n}
$$

with $n \neq 0,1,2$, that leads to consistent results with the truncation $\mathfrak{R e}(Z)=0$ (but not with $\mathfrak{I m}(Z)=0$ ). The model (4.1) is of course related to the one of section 3 by a complex rescaling of the $\chi^{\Lambda}$, and thus the truncations considered here and in the preceding section are actually two different truncations of the same model.

From (2.4), taking $\left|\chi^{0}\right|=1$ we obtain the Kähler potential

$$
e^{-\mathcal{K}}=\frac{i^{n}}{4(1-n)} Z^{1-n}[n Z+(2-n) \bar{Z}]+\text { c.c. },
$$

and, imposing $\mathfrak{R e}(Z)=0$, the Kähler metric

$$
\mathcal{G}=\left.\partial_{Z} \partial_{\bar{Z}} \mathcal{K}\right|_{\mathfrak{R e}(Z)=0}=-\frac{n(2-n)}{4} \mathfrak{I m}(Z)^{-2}=\frac{n_{0} n_{1}}{\left(n_{1}-n_{0}\right)^{2}} e^{\frac{n_{0}-n_{1}}{2} \phi}
$$

with $n_{0} \equiv-\frac{2 n}{1-n}, n_{1} \equiv \frac{2(2-n)}{1-n}=4-n_{0}, \phi \equiv \frac{4}{n_{1}-n_{0}} \log \mathfrak{I m}(Z)$.

From equation (2.6) one obtains the vectors' kinetic matrix

$$
\mathcal{N}=-\frac{i}{8}\left(\begin{array}{cc}
n_{0} e^{\frac{n_{1}}{2} \phi} & 0 \\
0 & n_{1} e^{\frac{n_{0}}{2} \phi}
\end{array}\right)
$$

while (2.8) leads to the scalar potential

$$
V=\frac{1}{2}\left[\frac{n_{0}\left(n_{0}-1\right)}{t_{0}^{2}} e^{-\frac{n_{1}}{2} \phi}+\frac{n_{1}\left(n_{1}-1\right)}{t_{1}^{2}} e^{-\frac{n_{0}}{2} \phi}\right],
$$

where we defined as before $t_{\Lambda} \equiv-\frac{n_{\Lambda}}{2 g C_{\Lambda}}$.

Substituting in eq. (2.7) we have

$$
\begin{aligned}
e^{-1} \mathcal{L}= & R+\frac{n_{0} n_{1}}{8} \partial_{\mu} \phi \partial^{\mu} \phi-\frac{n_{0}}{4} e^{\frac{n_{1}}{2} \phi} F_{\mu \nu}^{0} F^{0 \mu \nu}-\frac{n_{1}}{4} e^{\frac{n_{0}}{2} \phi} F_{\mu \nu}^{1} F^{1 \mu \nu} \\
& -\frac{1}{2}\left[\frac{n_{0}\left(n_{0}-1\right)}{t_{0}^{2}} e^{-\frac{n_{1}}{2} \phi}+\frac{n_{1}\left(n_{1}-1\right)}{t_{1}^{2}} e^{-\frac{n_{0}}{2} \phi}\right],
\end{aligned}
$$

which differs from the Lagrangian obtained in the previous section only by a sign in front of $n_{0}$ in the exponents and the absence of the cross term in the potential. To avoid ghost fields in the action we must restrict $n_{0}$ and $n_{1}$ to positive values, which corresponds to have in the prepotential either $n<0$ or $n>2$. 
(2.11), together with $\mathfrak{R e}(Z)=0$, leads to

$$
\begin{array}{cc}
\mathcal{I}_{0}=-\frac{n_{0}}{8} e^{\phi} \mathcal{I}^{1}, & \mathcal{I}_{1}=\frac{n_{1}}{8} e^{\phi} \mathcal{I}^{0}, \\
\mathcal{R}^{0}=e^{\frac{n_{0}-n_{1}}{4} \phi} \mathcal{I}^{1}, & \mathcal{R}^{1}=-e^{\frac{n_{1}-n_{0}}{4} \phi} \mathcal{I}^{0}, \\
\mathcal{R}_{0}=\frac{n_{0}}{8} e^{\frac{n_{1}}{2} \phi} \mathcal{I}^{0}, & \mathcal{R}_{1}=\frac{n_{1}}{8} e^{\frac{n_{0}}{2} \phi} \mathcal{I}^{1},
\end{array}
$$

as well as

$$
\frac{1}{2|X|^{2}}=\langle\mathcal{R} \mid \mathcal{I}\rangle=\frac{1}{2}\left[e^{\frac{n_{1}}{2} \phi}\left(\mathcal{I}^{0}\right)^{2}+e^{\frac{n_{0}}{2} \phi}\left(\mathcal{I}^{1}\right)^{2}\right]
$$

From (4.7) and (2.18) we see that, since we exclude the case $C_{0}=C_{1}=0, \mathcal{I}^{1}=0$ is equivalent to $C_{0}=0, \mathcal{I}^{0}=0$ is equivalent to $C_{1}=0$, and $C_{1} \mathcal{I}^{1}=-\frac{n_{1}}{n_{0}} C_{0} \mathcal{I}^{0}$.

\subsection{Construction of the solution}

As before we take

$$
W_{m}^{x}=\delta_{m}^{x}, \quad C_{\Lambda} \tilde{A}^{\Lambda}=C_{\Lambda} \mathcal{I}^{\Lambda}=0 .
$$

Since $C_{1} \mathcal{I}^{1}=-\frac{n_{1}}{n_{0}} C_{0} \mathcal{I}^{0}, C_{\Lambda} \mathcal{I}^{\Lambda}=0$ with $n_{0} \neq 2^{5}$ implies $C_{0} \mathcal{I}^{0}=0$. One has thus either $C_{0}=\mathcal{I}^{1}=0$ or $C_{1}=\mathcal{I}^{0}=0$. We will consider just the first case since the second can be obtained simply by exchanging 0 and 1 indices. We have thus

$$
C_{0}=0, \quad \mathcal{I}^{1}=\mathcal{I}_{0}=\mathcal{R}^{0}=\mathcal{R}_{1}=0,
$$

and from $C_{\Lambda} \tilde{A}^{\Lambda}=0$, taking into account that $C_{1} \neq 0$,

$$
\tilde{A}^{1}=0 \text {. }
$$

Eq. (2.17) yields

$$
\tilde{F}_{m n}^{0}=-\frac{1}{\sqrt{2}} \varepsilon_{m n p} \partial_{p} \mathcal{I}^{0}
$$

and from the Bianchi identity $d \tilde{F}^{0}=0$ we obtain

$$
\partial_{p} \partial_{p} \mathcal{I}^{0}=0 \quad \Rightarrow \quad \mathcal{I}^{0}=\sqrt{2} \mathcal{H}_{0},
$$

where $\mathcal{H}_{0}$ is a generic harmonic function of the spatial coordinates.

Using (4.10) and $C_{\Lambda} \tilde{A}^{\Lambda}=0$, from eq. (2.20) we conclude as before $\tilde{\omega}=d f$, where $f$ is a generic function of the spatial coordinates. (2.19) implies then

$$
\partial_{p} \partial_{p}\left(\tilde{\mathcal{I}}_{1}+\frac{g C_{1}}{2 \sqrt{2}} f\right)=0 \quad \Rightarrow \quad \tilde{\mathcal{I}}_{1}=\frac{n_{1}}{4 \sqrt{2}}\left(f / t_{1}+\mathcal{H}_{1}\right)
$$

\footnotetext{
${ }^{5}$ For $n_{0}=2(n \rightarrow \pm \infty)$ we could take both $C_{0}, C_{1} \neq 0$ (equivalently, $\mathcal{I}^{0}, \mathcal{I}^{1} \neq 0$ ); however this would lead to exactly the same solution we obtain here, with just a field redefinition.
} 
where $\mathcal{H}_{1}$ is another harmonic function of the spatial coordinates. Using (2.18) and (4.7) one gets

$$
\mathcal{I}_{1}=\frac{n_{1}}{4 \sqrt{2}}\left((\tau+f) / t_{1}+\mathcal{H}_{1}\right), \quad e^{\phi}=\frac{(f+\tau) / t_{1}+\mathcal{H}_{1}}{\mathcal{H}_{0}}
$$

and from (4.8) one computes

$$
\frac{1}{2|X|^{2}}=\left(\frac{\tau+f}{t_{1}}+\mathcal{H}_{1}\right)^{\frac{n_{1}}{2}} \mathcal{H}_{0}^{\frac{n_{0}}{2}}
$$

Eliminating $f$ by introducing the new time coordinate $t=\tau+f$, the solution can be written as

$$
\begin{gathered}
d s^{2}=\mathcal{U}^{-2} d t^{2}-\mathcal{U}^{2} d \vec{y}^{2} \\
F^{0}=-\frac{1}{2} \varepsilon_{m n p} \partial_{p} \mathcal{H}_{0} d y^{m} \wedge d y^{n}, \quad A^{1}=\left(\frac{t}{t_{1}}+\mathcal{H}_{1}\right)^{-1} d t \\
\phi=\ln \left(\frac{t / t_{1}+\mathcal{H}_{1}}{\mathcal{H}_{0}}\right)
\end{gathered}
$$

with

$$
\mathcal{U} \equiv\left(\frac{t}{t_{1}}+\mathcal{H}_{1}\right)^{\frac{n_{1}}{4}} \mathcal{H}_{0}^{\frac{n_{0}}{4}}
$$

This is, with the right choice for $\mathcal{H}_{0}$ and $\mathcal{H}_{1}$, the spacetime found in [17] and discussed further in [11]; however in this case, instead of having two gauge fields in an electric configuration, one of them is magnetic due to the different sign in the exponent of its scalar coupling. In other words, one of the field strengths in the Gibbons-Maeda solution is dualized here.

\section{$5 \quad$ Final remarks}

Let us conclude our paper with the following suggestions for possible extensions and questions for future work:

- Add rotation. This is under investigation [26].

- Construct the corresponding 'nonextremal' solution (i.e., the one that does not admit fake Killing spinors), which might be of astrophysical relevance.

- Do our solutions allow to study dynamical processes like black hole collisions, similar to what was done in $[6,27]$ for the Kastor-Traschen spacetime? 
- Does the attractor mechanism [28-32] continue to work in the time-dependent case? This issue has not been addressed in the literature so far.

- One may consider more general Gauduchon-Tod base spaces and/or more complicated prepotentials in the construction of [15], and see whether this leads to physically interesting solutions.

\section{References}

[1] G. C. McVittie, "The mass-particle in an expanding universe," Mon. Not. Roy. Astron. Soc. 93 (1933) 325.

[2] B. C. Nolan, "A point mass in an isotropic universe: Existence, uniqueness and basic properties," Phys. Rev. D 58 (1998) 064006 [gr-qc/9805041].

[3] B. C. Nolan, "A point mass in an isotropic universe. 2. Global properties," Class. Quant. Grav. 16 (1999) 1227.

[4] N. Kaloper, M. Kleban and D. Martin, "McVittie's legacy: Black holes in an expanding universe," Phys. Rev. D 81 (2010) 104044 [arXiv:1003.4777 [hep-th]].

[5] J. Sultana and C. C. Dyer, "Cosmological black holes: A black hole in the Einstein-de Sitter universe," Gen. Rel. Grav. 37 (2005) 1347.

[6] D. Kastor and J. H. Traschen, "Cosmological multi-black hole solutions," Phys. Rev. D 47 (1993) 5370 [hep-th/9212035].

[7] D. Klemm and W. A. Sabra, "Charged rotating black holes in 5-D Einstein-Maxwell (A)dS gravity," Phys. Lett. B 503 (2001) 147 [hep-th/0010200].

[8] D. Klemm and W. A. Sabra, "General (anti-)de Sitter black holes in five-dimensions," JHEP 0102 (2001) 031 [hep-th/0011016].

[9] K. -i. Maeda, N. Ohta and K. Uzawa, "Dynamics of intersecting brane systems -Classification and their applications-," JHEP 0906 (2009) 051 [arXiv:0903.5483 [hep-th]].

[10] K. -i. Maeda and M. Nozawa, "Black hole in the expanding universe from intersecting branes," Phys. Rev. D 81 (2010) 044017 [arXiv:0912.2811 [hep-th]].

[11] K. -i. Maeda and M. Nozawa, "Black hole in the expanding universe with arbitrary power-law expansion," Phys. Rev. D 81 (2010) 124038 [arXiv:1003.2849 [gr-qc]].

[12] K. -i. Maeda and M. Nozawa, "Black hole solutions in string theory," Prog. Theor. Phys. Suppl. 189 (2011) 310 [arXiv:1104.1849 [hep-th]].

[13] M. Nozawa and K. -i. Maeda, "Cosmological rotating black holes in five-dimensional fake supergravity," Phys. Rev. D 83 (2011) 024018 [arXiv:1009.3688 [hep-th]]. 
[14] D. Kastor and J. H. Traschen, "Particle production and positive energy theorems for charged black holes in De Sitter," Class. Quant. Grav. 13 (1996) 2753 [gr-qc/9311025].

[15] P. Meessen and A. Palomo-Lozano, "Cosmological solutions from fake $N=2$ EYM supergravity," JHEP 0905 (2009) 042 [arXiv:0902.4814 [hep-th]].

[16] J. B. Gutowski and W. A. Sabra, "HKT geometry and fake five-dimensional supergravity," Class. Quant. Grav. 28 (2011) 175023 [arXiv:1009.4453 [hep-th]].

[17] G. W. Gibbons and K. -i. Maeda, "Black holes in an expanding universe," Phys. Rev. Lett. 104 (2010) 131101 [arXiv:0912.2809 [gr-qc]].

[18] P. Gauduchon and P. K. Tod, "Hyper-Hermitian metrics with symmetry", J. Geom. Phys. 25 (1998) 291.

[19] K. Behrndt and M. Cvetič, "Time dependent backgrounds from supergravity with gauged noncompact R symmetry," Class. Quant. Grav. 20 (2003) 4177 [hep-th/0303266].

[20] C. W. Misner and D. H. Sharp, "Relativistic equations for adiabatic, spherically symmetric gravitational collapse," Phys. Rev. 136 (1964) B571.

[21] S. A. Hayward, "General laws of black hole dynamics," Phys. Rev. D 49 (1994) 6467.

[22] S. A. Hayward, "Unified first law of black hole dynamics and relativistic thermodynamics," Class. Quant. Grav. 15 (1998) 3147 [gr-qc/9710089].

[23] S. A. Hayward, R. Di Criscienzo, L. Vanzo, M. Nadalini and S. Zerbini, "Local Hawking temperature for dynamical black holes," Class. Quant. Grav. 26 (2009) 062001 [arXiv:0806.0014 [gr-qc]].

[24] S. W. Hawking and G. F. R. Ellis, "The large scale structure of space-time," Cambridge University Press, Cambridge, 1973.

[25] V. A. Kostelecký and M. J. Perry, "Solitonic black holes in gauged $N=2$ supergravity," Phys. Lett. B 371 (1996) 191 [hep-th/9512222].

[26] S. Chimento and D. Klemm, to appear.

[27] D. R. Brill, G. T. Horowitz, D. Kastor and J. H. Traschen, "Testing cosmic censorship with black hole collisions," Phys. Rev. D 49 (1994) 840 [gr-qc/9307014].

[28] S. Ferrara, R. Kallosh and A. Strominger, " $N=2$ extremal black holes," Phys. Rev. D 52 (1995) 5412 [arXiv:hep-th/9508072].

[29] A. Strominger, "Macroscopic entropy of $N=2$ extremal black holes," Phys. Lett. B 383 (1996) 39 [arXiv:hep-th/9602111].

[30] S. Ferrara and R. Kallosh, "Supersymmetry and attractors," Phys. Rev. D 54 (1996) 1514 [arXiv:hep-th/9602136]. 
[31] S. Ferrara and R. Kallosh, "Universality of supersymmetric attractors," Phys. Rev. D 54 (1996) 1525 [arXiv:hep-th/9603090].

[32] S. Ferrara, G. W. Gibbons and R. Kallosh, "Black holes and critical points in moduli space," Nucl. Phys. B 500 (1997) 75 [arXiv:hep-th/9702103]. 